\title{
Comments on some recent measurements of anomalously steep N2O and O3tracer spectra in the stratospheric surf zone
}

Article

Published Version

Ngan, K. and Shepherd, T. G. (1997) Comments on some recent measurements of anomalously steep N2O and O3tracer spectra in the stratospheric surf zone. Journal of Geophysical Research, 102 (D20). pp. 24001-24004. ISSN 0148-0227 doi: https://doi.org/10.1029/97JD01767 Available at https://centaur.reading.ac.uk/32864/

It is advisable to refer to the publisher's version if you intend to cite from the work. See Guidance on citing.

Published version at: http://dx.doi.org/10.1029/97JD01767

To link to this article DOI: http://dx.doi.org/10.1029/97JD01767

Publisher: American Geophysical Union

All outputs in CentAUR are protected by Intellectual Property Rights law, including copyright law. Copyright and IPR is retained by the creators or other copyright holders. Terms and conditions for use of this material are defined in the End User Agreement.

www.reading.ac.uk/centaur 
Central Archive at the University of Reading

Reading's research outputs online 


\title{
Comments on some recent measurements of anomalously steep $\mathrm{N}_{2} \mathrm{O}$ and $\mathrm{O}_{3}$ tracer spectra in the stratospheric surf zone
}

\author{
Keith Ngan and Theodore G. Shepherd \\ Department of Physics, University of Toronto, Toronto, Canada
}

\begin{abstract}
Recent aircraft measurements, primarily in the extratropics, of the horizontal variance of nitrous oxide $\left(\mathrm{N}_{2} \mathrm{O}\right)$ and ozone $\left(\mathrm{O}_{2}\right)$ in the middle stratosshere indicate that horizontal spectra of the tracer variance scale nearly as $k^{-2}$, where $k$ is the spatial wavenumber along the aircraft flight track [Strahan and Mahlman, 1994; Bacmeister et al., 1996]. This spectral scaling has been regarded as inconsistent with the accepted picture of stratospheric tracer motion; large-scale quasi-twodimensional tracer advection typically yields a $k^{-1}$ scaling (i.e., the classical Batchelor spectrum). In this paper it is argued that the nearly $k^{-2}$ scaling seen in the measurements is a natural outcome of quasi-two-dimensional filamentation of the polar vortex edge. The accepted picture of stratospheric tracer motion can thus be retained: no additional physical processes are needed to account for deviations from the Batchelor spectrum. Our argument is based on the finite lifetime of tracer filaments and on the "singularity spectrum" associated with a one-dimensional field composed of randomly spaced jumps in concentration.
\end{abstract}

\section{Introduction}

It is now generally accepted that on timescales of less than 2 weeks or so, tracer motion in the lower and middle stratosphere is dominated by the largescale quasi-two-dimensional velocity field [Waugh and Plumb, 1994; Norton, 1994]. Many studies have shown that the development of small-scale filamentary structure in tracer fields (on isentropic surfaces) can be successfully explained in terms of breaking planetary waves and the emergence of an accompanying "surf zone" [e.g., McIntyre and Palmer, 1983; Juckes and McIntyre, 1987]. Because of vertical shearing of the horizontal flow, horizontal filaments on different isentropic surfaces form sloping sheets of tracer [Balluch and Haynes, 1997; Haynes and Anglade, 1997], which are manifested in the vertical as laminae in concentration profiles [Orsolini, 1995; Newman and Schoeberl, 1995; Mariotti et al., 1997].

At first sight, this picture of stratospheric tracer motion appears inconsistent with the recent aircraft measurements of $\mathrm{N}_{2} \mathrm{O}$ and $\mathrm{O}_{3}$ tracer spectra in the stratospheric surf zone described by Strahan and Mahlman [1994] and Bacmeister et al. [1996]. In a range of scales between approximately 1 and $1000 \mathrm{~km}$ a nearly $k^{-2}$ power spectrum has been found. (Actually, the spectra lie between $k^{-5 / 3}$ and $k^{-2}$; differences between

Copyright 1997 by the American Geophysical Union.

Paper number 97JD01767.

0148-0227/97/97JD-01767\$09.00 these exponents are hard to discern.) By contrast, twodimensional homogeneous turbulence theory predicts a $k^{-1}$ tracer spectrum for a dynamical regime in which large-scale strain is dominant and diffusion is negligible [Lesieur, 1990].

In attempting to resolve this discrepancy it has been suggested that processes other than quasi-horizontal large-scale advection play a role: enhanced dissipation and diffusion are mentioned in Strahan and Mahlman [1994], and gravity waves in Bacmeister et al. [1996]. However, as noted in the latter reference, these processes are incompatible with the existence of widespread filamentary structure.

These "anomalous" spectra are not artifacts of the aircraft measurements: a $k^{-2}$ spectral scaling has also been found in the Total Ozone Mapping Spectrometer (TOMS) data set [Sirovich et al., 1995]. This suggests that these spectra do reflect fundamental dynamical processes in the stratosphere; the question, of course, is which ones.

\section{The Singularity Spectrum}

We wish to point out that the measurements can be reconciled with the current picture of stratospheric tracer motion if one recognizes the patchiness of the tracer distribution. Along-flight-track profiles of tracer concentration exhibit a sequence of sharply defined jumps, the result being a set of "cliff-like" features [Strahan and Mahlman, 1994; Waugh et al., 1994; Plumb et al., 1994]. It is well known that a one-dimensional 
field containing a discontinuous jump will have a power spectrum of $k^{-2}$; more generally, a series of jumps of random amplitude spaced at random intervals will have a $k^{-2}$ spectrum for scales small in comparison with the characteristic spacing between the jumps but large in comparison with the thickness of an individual jump [e.g., Saffman, 1971]. Such structures can be generated by the filamentation of a single interface. In the stratosphere, the edge of the polar vortex is a sharp interface between chemically distinct air masses [Schoeberl et al., 1992; Bowman and Mangus, 1993], and filamentation of the vortex edge is a well-documented phenomenon [Polvani and Plumb, 1992; Waugh and Plumb, 1994; Norton, 1994; Waugh et al., 1994; Plumb et al.,

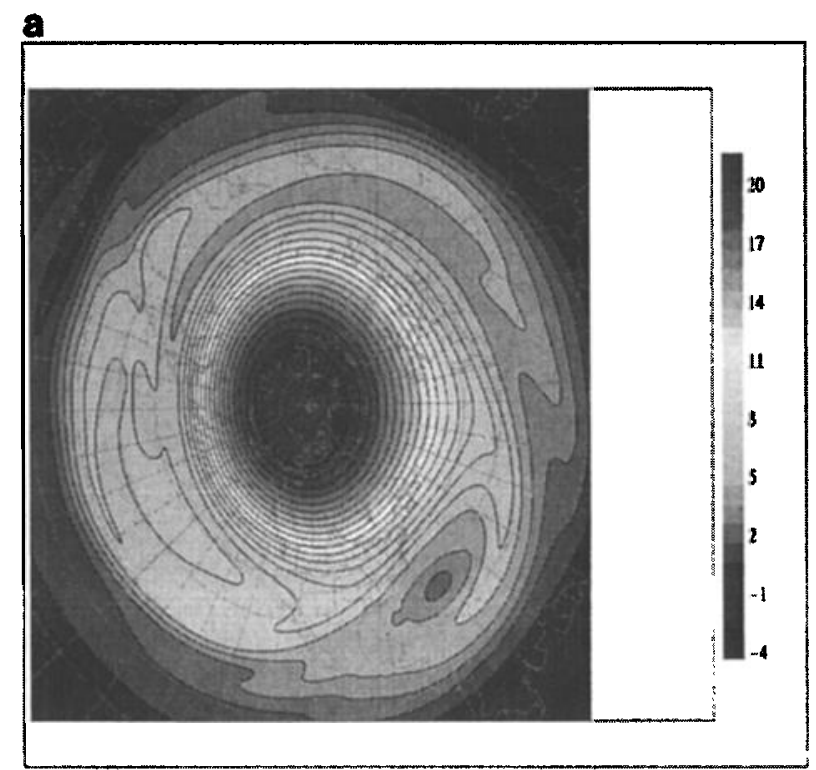

b

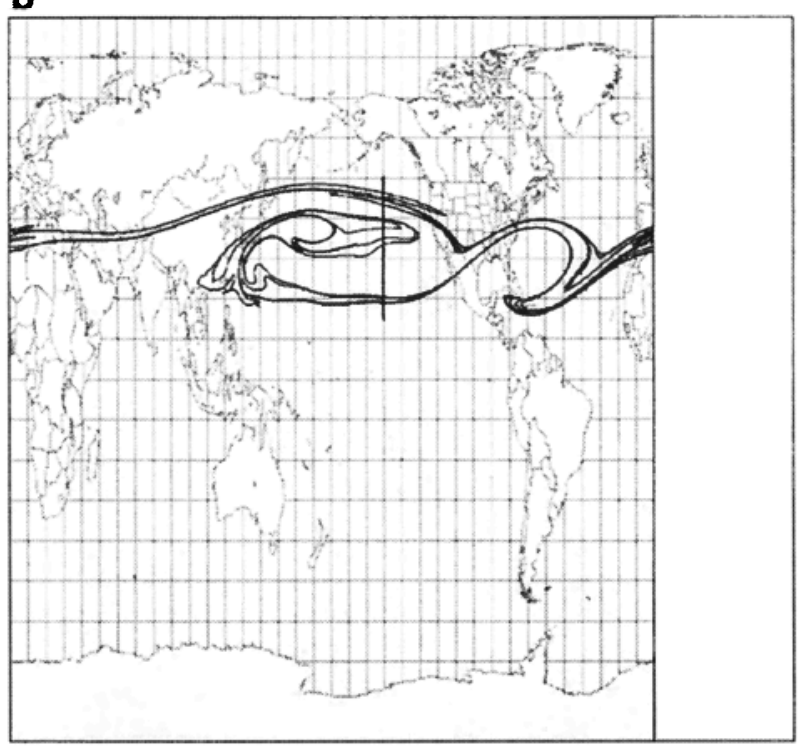

1994]. A sharp chemical interface is, furthermore, observed at the subtropical edge of the surf zone [Fahey et al., 1996], and filamentation of this interface [Randel et al., 1993; Waugh, 1993] may provide another source of cliff-like tracer structures.

This explanation makes no assumptions about the nature of the velocity field at small scales; it relies on the dominant role of the large-scale (quasi-two-dimensional) velocity field. For this reason it does not contradict the hypothesis that the spectral scalings of dynamical fields such as velocity and potential temperature are, at small scales, largely determined by gravity wave motions [Bacmeister et al., 1996].

We illustrate how such a $k^{-2}$ singularity spectrum
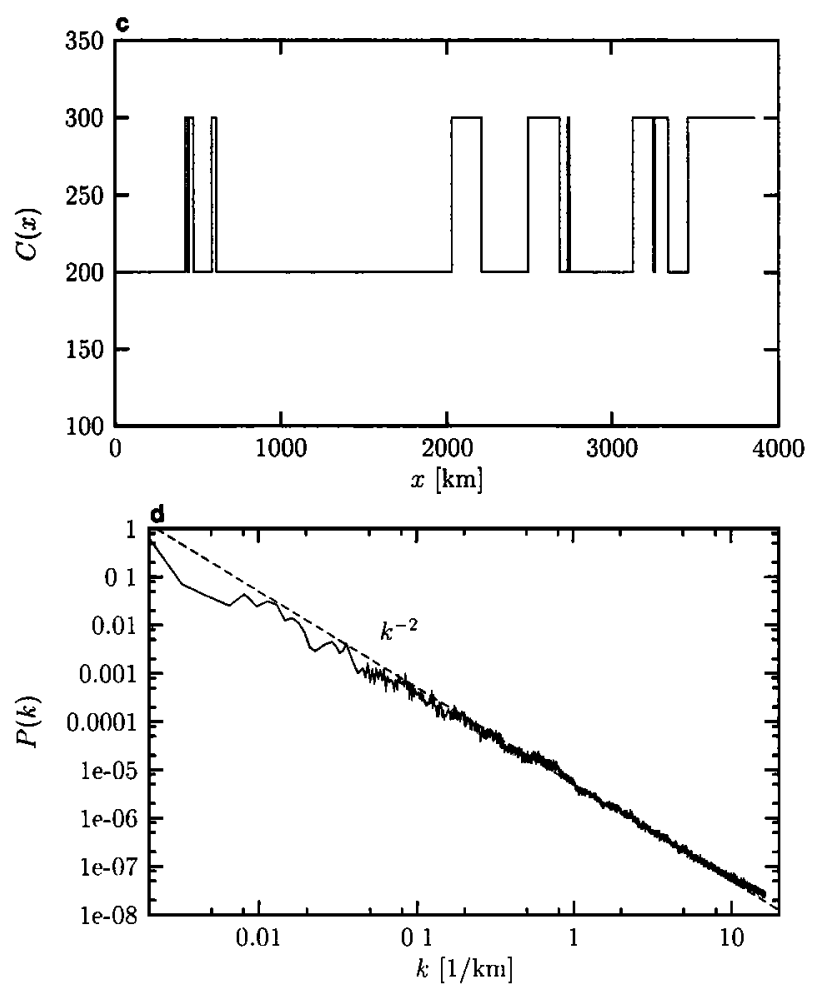

Figure 1. One-dimensional power spectrum associated with a material contour separating chemically distinct air masses. The evolution of the contour is determined using winds from a shallow-water model with stationary forcing; this kind of model has been successfully used to simulate the dynamics of a perturbed polar vortex. (a) The potential vorticity distribution after the onset of Rossby-wave breaking. Units are in $10^{-9} \mathrm{~m}^{-1} \mathrm{~s}^{-1}$. (b) A material line initially coinciding with the "vortex edge", which has been evolved using a contour advection scheme [Schoeberl and Newman, 1995]. A onedimensional cut (the thick line) through this material line is also shown. (c) A nominal concentration profile, sampling either vortex or midlatitude air, that corresponds to the one-dimensional cut. The length of the cut is approximately the same as the length of the aircraft flights. (d) An averaged power spectrum for an ensemble of 1000 cuts centered around the one shown in Figure 1b; essentially identical results have been obtained with different material contours and different ensembles. A Welch window is used in computing the periodogram. 
can arise from the filamentation of a single interface. Figure la shows the shallow-water potential vorticity for a simulated polar vortex disturbed by stationary planetary wave forcing. A material contour marking the polar vortex edge is shown in Figure 1b. A horizontal cut through this contour-which is the analog of an aircraft flight path-is indicated, and a nominal chemical concentration profile along the cut is plotted in Figure 1c. The cliff-like structure of this profile is strongly reminiscent of that seen in the aircraft measurements. Figure 1d, an average over cuts taken around the one shown in Figure 1c, confirms that the associated power spectrum exhibits $k^{-2}$ scaling.

The analyses of Bacmeister et al. [1996] and Strahan and Mahlman [1994] differ in that the former compute spectra for flight segments of length $\sim 200 \mathrm{~km}$, while the latter compute spectra for flight legs of length $\sim 1000 \mathrm{~km}$. Most of the flight segments in the former case presumably do not contain cliff-like structures; however, the ensemble averaged spectrum should be dominated by those that do, and a $k^{-2}$ spectrum is thus to be expected. This has been verified for the idealized calculation of Figure 1.

In the stratosphere, of course, the creation and destruction of vortex filaments coexist in some kind of forced-dissipative equilibrium, which cannot be captured by an initial-value calculation. Nevertheless, the calculation is representative of the stratospheric surf zone in the same way (and for the same reasons) that finite-time contour advection calculations are successful in replicating real filamentary structure [Wough et al., 1994; Plumb et al., 1994]; both rely on the existence of a sharp chemical edge and the finite lifetime of individual tracer filaments.

\section{Discussion}

The existence of a sharp chemical edge separating two regions of distinct tracer concentration is a reasonable assumption during the fall, winter, and spring, which is when most of the measurements took place. During the summer, when the polar vortex breaks down, this argument no longer holds, and different spectral scalings would then be expected.

The singularity spectrum argument requires the jumps to be well separated. Different scalings are possible if this condition is not satisfied. The power spectrum $P(k)$ along the cut is more generally given by [Vassilicos and Hunt, 1991]

$$
P(k) \sim k^{-2+D_{K}},
$$

where $D_{K}$ is the Kolmogorov capacity (box-counting dimension) of the interfacial intersection points and $0 \leq D_{K}<1$. In the case shown in Figure $1, D_{K} \approx 0$, so $P(k) \approx k^{-2}$. In the opposite limit, $D_{K} \rightarrow 1$, the filament is space filling, and one recovers $P(k) \sim k^{-1}$; this situation is realized in the long-time nondiffusive limit when there is persistent random strain, such as is as- sumed in Batchelor's original derivation of the $k^{-1}$ spectrum [Batchelor, 1959]. (An asymptotic $k^{-1}$ spectrum has also been obtained in the long-time limit for weakly diffusive flows exhibiting chaotic advection [Pierrehumbert, 1994; Antonsen et al., 1996].)

In the aircraft measurements, tracer concentration profiles do indeed contain well-separated jumps. This is consistent with current estimates of filament lifetimes and stretching rates in the middle stratosphere. Lifetimes of passive filaments (which are limited by vertical diffusion) are estimated to be around 2 weeks [Balluch and Haynes, 1997; Haynes and Anglade, 1997]; lifetimes of photochemically reacting ozone filaments (in the northern surf zone) are similar [von der Gathen et al., 1995]; and stretching rates around $0.2 \mathrm{day}^{-1}$ are typical [Schoeberl and Newman, 1995]. These estimates suggest that tracer filaments are destroyed before they have time to fill the surf zone or develop significant fractal structure. A tracer spectrum that is clearly distinct from $k^{-1}$ and close to, though probably not coinciding with, $k^{-2}$ would then be expected for both $\mathrm{N}_{2} \mathrm{O}$ and $\mathrm{O}_{3}$.

Acknowledgments. The authors thank S. Gravel and M. Roch of Recherche en prévision numérique (AES - Dorval) for providing the shallow-water model, P. H. Haynes for a careful reading of the manuscript, and $R$. C. Bailey and J. N. Koshyk for helpful comments. This paper is based on research partially carried out at the Isaac Newton Institute for Mathematical Sciences, Cambridge, UK during the programme "Mathematics of Atmosphere and Ocean Dynamics", July-December 1996, and is Newton Institute publication number NI97012-AOD. Additional support was provided by the Natural Sciences and Engineering Research Council and the Atmospheric Environment Service of Canada.

\section{References}

Antonsen, T. M., Z. Fan, E. Ott, and E. Garcia-Lopez, The role of chaotic orbits in the determination of power spectra of passive scalars, Phys. Fluids, 8, 3094-3104, 1996.

J. T. Bacmeister, S. D. Eckermann, P. A. Newman, L. Lait, K. R. Chan, M. Loewenstein, M. H. Proffitt, and B. L. Gary 1996 Stratospheric horizontal wavenumber spectra of winds, potential temperature, and atmospheric tracers observed by high-altitude aircraft, J. Geophys. Res., 101, 9441-9470, 1996.

Balluch, M. G., and P. H. Haynes, Quantification of lower stratospheric mixing processes using aircraft data, J. Geophys. Res., in press, 1997.

Batchelor, G. K., Small-scale variation of convected quantities like temperature in turbulent fluid, 1, General discussion and the case of small conductivity, J. Fluid Mech., 5, 113-133, 1959.

Bowman, K. P., and N. J. Mangus, Observations of deformation and mixing of the total ozone field in the Antarctic polar vortex, J. Atmos. Sci., 50, 2915-2921, 1993.

Fahey, D. W., et al., In situ observations of $\mathrm{NO}_{y}, \mathrm{O}_{3}$, and the $\mathrm{NO}_{y} / \mathrm{O}_{3}$ ratio in the lower stratosphere, Geophys. Res. Lett., 23, 1653-1656, 1996.

Haynes, P. H., and J. Anglade, The vertical-scale cascade in atmospheric tracers due to large-scale differential advection, J. Atmos. Sci., 54, 1121-1136, 1997. 
Juckes, M. N., and M. E. McIntyre, A high-resolution onelayer model of breaking planetary waves in the stratosphere, Nature, 328, 590-596, 1987.

Lesieur, M., Turbulence in Fluids, 2nd ed., Kluwer Acad, Norwell, Mass., 1990.

Mariotti, A., M. Moustaoui, B. Legras, and H. Teitelbaum, Comparison between vertical ozone soundings and reconstructed potential vorticity maps by contour advection with surgery, J. Geophys. Res., 102, 6131-6142, 1997.

McIntyre, M. E., and T. N. Palmer, Breaking planetary waves in the stratosphere, Nature, 305, 593-600, 1983.

Newman, P. A., and M. R. Schoeberl, A reinterpretation of the data from the NASA stratosphere-troposphere exchange project, Geophys. Res. Lett., 22, 2501-2504, 1995.

Norton, W. A., Breaking Rossby waves in a model stratosphere diagnosed by a vortex-following coordinate system and a technique for advecting material contours, J. Atmos. Sci., 51, 654-673, 1994.

Orsolini, Y. J., On the formation of ozone laminae at the edge of the Arctic polar vortex, Q. J.R. Meteorol. Soc., 528, 1923-1941, 1995.

Pierrehumbert, R. T., Tracer microstructure in the largeeddy dominated regime, in Chaos Applied to Fluid Mixing, edited by $\mathrm{H}$. Aref and M. S. El Naschie, Pergamon, Tarrytown, N.Y. 1994.

Plumb, R. A., D. W. Waugh, R. J. Atkinson, P. A. Newman, L. R. Lait, M. R. Schoeberl, E. V. Browell, A. J. Simmons, and $M$. Lowenstein, Intrusions into the lower stratospheric Arctic vortex during the winter of 1991-1992, J. Geophys. Res., 99, 1089-1105, 1994.

Polvani, L. M., and R. A. Plumb, Rossby wave breaking, microbreaking, filamentation and secondary vortex formation: The dynamics of a perturbed vortex, J. Atmos. Sci., 49, 462-476, 1992.

Randel, W. J., et al., Stratospheric transport from the tropics to middle latitudes by planetary-wave mixing, Nature, 365, 533-535, 1993.

Saffman, P. G., On the spectrum and decay of random twodimensional vorticity distributions at large Reynolds number, Stud. Appl. Math., 50, 377-383, 1971.
Schoeberl, M. R., L. R. Lait, P. A. Newman, and J. A. Rosenfield, The structure of the polar vortex, J. Geophys. Res., 97, 7859-7882, 1992.

Schoeberl, M. R., and P. A. Newman, A multiple-level trajectory analysis of vortex filaments, J. Geophys. Res., 100, 25,801-25,815, 1995.

Sirovich, L., R. Everson, and D. Manin, Turbulent spectrum of the Earth's ozone field, Phys. Rev. Lett., 74, 2611-2614, 1994.

Strahan, S. E., and J. D. Mahlman, Evaluation of the SKYHI general circulation model using aircraft $\mathrm{N}_{2} \mathrm{O}$ measurements, 2, Tracer variability and diabatic meridional circulation, J. Géophys. Res., 99, 10,319-10,332, 1994.

Vassilicos, J. C., and J. C. R. Hunt, Fractal dimensions and spectra of interfaces with application to turbulence, Proc. R. Soc. London A, 435, 505-534, 1991.

von der Gathen, P., et al., Observational evidence for chemical ozone depletion over the Arctic in winter 1991-92, Nature, 375, 131-134, 1995.

Waugh, D. W., Subtropical stratospheric mixing linked to disturbances in the polar vortices, Nature, $365,535-537$, 1993.

Waugh, D. W., and R. A. Plumb, Contour advection with surgery: A technique for investigating fine-scale structure in tracer transport, J. Atmos. Sci., 51, 530-540, 1994.

Waugh, D. W., et al., Transport out of the lower stratospheric Arctic vortex by Rossby wave breaking, J. Geophys. Res., 99, 1071-1088, 1994.

K. Ngan and T. G. Shepherd, Department of Physics, University of Toronto, $60 \mathrm{St}$. George Street, Toronto, Ontario M5S 1A7, Canada. (e-mail: keith@ atmosp.physics.utoronto.ca; tgs@atmosp.physics. utoronto.ca)

(Received February 11, 1997; revised June 10, 1997; accepted June 13, 1997.) 